\title{
CARACTERIZAÇÃO MORFOAGRONÔMICA DE GERMOPLASMA DE AÇAIZEIRO NO NORDESTE PARAENSE ${ }^{1}$
}

\author{
ROSEMIRO DOS SANTOS GALATE², MILTON GUILHERME DA COSTA MOTA², \\ JOSÉ MARIA DEMETRIO GAIA ${ }^{4}$, MERILENE DO SOCORRO SILVA COSTA ${ }^{5}$
}

RESUMO - A caracterização morfológica de germoplasma é requisito básico para determinar e quantificar a variabilidade genética de germoplasma. A eliminação de descritores redundantes reduz o trabalho de tomada de dados, sem ocasionar redução na precisão da caracterização. Neste trabalho, objetivou-se analisar e quantificar a variabilidade fenotípica de matrizes de açaizeiro no nordeste paraense, analisando a importância relativa de diferentes características morfológicas para variância fenotípica. Foram analisados 22 caracteres morfométricos, sendo cinco da planta (NEP, AE, NF, CAP e CEN), seis dos frutos (DLF, DTF, PF, PS, PP e RPF) e onze agronômicos (PC, PFC, RFC, NRC, NFR, NFC, NCP, PCF, CIC, CC e PTF). Utilizou-se a análise de componentes principais e os critérios de Jolliffe, Mardia e Cury para descarte de variáveis redundantes assistidos pelas correlações de Pearson. Necessitou-se de oito caracteres para explicar $80 \%$ da variação fenotípica. Sugeriram-se para descarte os caracteres PF, PFC, NFC, RPF, PCF, PTF, DTF e NFR. A dispersão gráfica 3D formou um grupo homogêneo e parcialmente disperso, permitindo detectar os genótipos EO-018 e EO-035 como os mais divergentes. Concluiu-se que há variabilidade para discriminar todas as matrizes, com $83,84 \%$ da variância fenotípica total, sendo 14 caracteres utilizados para este objetivo. Os critérios de descarte indicaram que se podem utilizar apenas os caracteres NEP, AE, CAP, CEN, NF, DLF, PS, PP, PC, RFC, NRC, CC, CIC e NCP para discriminar as matrizes, com perda mínima de informação. Pela análise gráfica 3D, observam-se matrizes divergentes, importantes para aumentar a variabilidade genética da coleção e o desenvolvimento de genótipos superiores.

Termos para indexação: Euterpe oleracea Mart, recursos genéticos, açaí, análise multivariada.

\section{MORPHOAGRONOMIC CHARACTERIZATION OF ASSAI PALM'S GERMPLASM FROM EASTERN AMAZON}

\begin{abstract}
The morphologic characterization is a basic step to analyze and quantify the germplasm genetic variability. The elimination of redundant descriptors reduces the labor for data collecting, without reduction of the precision of the characterization. This study aimed to analyze and quantify the phenotypic variability of assai palm's mother plant in the Northeast of Pará, Brazil, analyzing the relative importance of different morphological characteristics to phenotypic variance. Twenty two characters were analyzed: five from the plant (NEP; AE, NF; CAP and CEN); six from the fruits (DLF; DTF; PF; PS; PP and RPF); and eleven agronomics (PC; PFC; RFC; NRC; NFR; NFC; NCP; PCF; CIC; CC; and PTF). It was used the principal components analysis and Jolliffe, Mardia and Cury's criterions for discarding of variables assisted by the Pearson's correlations. It was necessary eight characters for explain $80 \%$ on the variation. It was suggested discarding the traits PF, PFC, NFC, RPF, PCF, PTF, DTF e NFR. The graphic dispersion formed a compact homogeneous group and partiality disperses in the flanks. The EO-018 e EO-035 genotypes were the most divergent. It was observed variability for genotype discrimination, with $83.84 \%$ on the characters. The use of the discarding criterions admitted the traits NEP, AE, CAP, CEN, NF, DLF, PS, PP, PC, RFC, $\mathrm{NRC}, \mathrm{CC}, \mathrm{CIC}$ e NCP for genotypes discrimination, with minimal lack of information. Graphic dispersion shows genotype divergence, important for to increase the genetic variability of collection and for production of superior genotypes.
\end{abstract}

Index terms: Euterpe oleracea Mart, genetic resources, grape, multivariate analysis.

\footnotetext{
${ }^{1}$ (Trabalho 263-11). Recebido em: 07-11-2011. Aceito para publicão em: 09-05-2012.

${ }^{2}$ Eng. Agr. MSc. Prof. UFRA-ICIBE (Instituto Ciberespacial), Av. Tancredo Neves s/n, C. Postal 917, CEP 66.077-530 Belém-PA, e-mail :rosemiro.galate@ufra.edu.br

${ }^{3}$ Eng. Agr. DSc. Prof. UFRA-ICIBE (Instituto Ciberespacial), Av. Tancredo Neves s/n, C. Postal 917, CEP 66.077-530 Belém-PA, e-mail: mota@amazon.com.br

${ }^{4}$ Eng. Agr. DSc. Bolsista DCR, CNPq-FAPESPA, UFRA, Av. Tancredo Neves s/n, C. Postal 917, CEP 66.077-530 Belém-PA, e-mail <josegaia@globo.com

${ }^{5}$ Eng. Flo. DSc. Prof. UFRA-ICIBE (Instituto Ciberespacial), Av. Tancredo Neves s/n, C. Postal 917, CEP 66.077-530 Belém-PA, e-mail: merilene@hotmail.com
} 


\section{INTRODUÇÃO}

O açaizeiro (Euterpe oleracea) distribui-se do litoral Atlântico Norte, desde o Maranhão até os arredores de Parintins, ao Sul do Rio Amazonas, em áreas permanentes (igapós) ou temporariamente inundáveis (várzeas baixas) (MENEZES NETO et al., 1995; CARVALHO et al., 1998; GONÇALVES et al., 2004). A divulgação dos benefícios nutricionais do açaí induziu a expansão do mercado de fruto e polpa. A área colhida vem aumentando, possivelmente, devido ao aumento de área cultivada e manejo de açaizais espontâneos, contribuindo para que o açaí lidere o mercado da fruticultura nacional, exportando, atualmente, 500 mil toneladas/ano (ROGEZ, 2000; SANTANA et al., 2008).

A caracterização morfológica e agronômica de germoplasma é requisito básico para determinar a variabilidade genética de germoplasma. Em espécies perenes, os descritores morfoagronômicos têm sido utilizados sem o conhecimento de sua contribuição real para a variabilidade (PEREIRA et al., 1992; DIAS et al., 1997). O elevado número de descritores tende a causar redundância devido à associação entre caracteres (DAHER et al., 1997). A eliminação de descritores redundantes é vantajosa, pois reduz o trabalho de tomada de dados, sem ocasionar redução na precisão da caracterização (PEREIRA et al., 1992). Vários são os procedimentos adotados para descarte de caracteres de baixo conteúdo informativo, como os utilizados por Daher et al. (1997), Dias et al. (1997), Strapasson et al. (2000), Alves et al. (2003) e Oliveira et al. (2006).

Estes últimos autores utilizaram 87 acessos de açaizeiro, com base em 28 descritores do Banco de Germoplasma da Embrapa Amazônia Oriental, provenientes de 21 municípios dos Estados do Pará, Maranhão e Amapá, entretanto nenhum estudo sobre a caracterização e divergência genética foi encontrado sobre matrizes procedentes dos municípios de Belém, incluindo as Ilhas de Mosqueiro e Combu, Capitão Poço, Ourém, Santarém Novo, Salinópolis e São João de Pirabas, todos situados no nordeste paraense. Vale ressaltar que a produção nativa do açaí da Ilha do Combu abastece praticamente todo o consumo da cidade de Belém

A utilização de técnicas multivariadas para análise dos caracteres morfoagronômicos de germoplasma é a mais apropriada, porque trata dos dados em sua multidimensionalidade. Um dos métodos mais empregados é a análise de componentes principais (ACP). Com base nesta análise, é possível quantificar a importância relativa de cada um dos caracteres para a quantificação da variabilidade genética, buscando, assim, a identificação daqueles com maior conteúdo informativo para a caracterização e a avaliação de germoplasma, como também disponibilizar a informação para rejeitar caracteres que quase não contribuem para a variação total (CRUZ et al., 2004). Objetivou-se, neste trabalho, analisar e quantificar a variabilidade fenotípica de matrizes de açaizeiro no nordeste paraense, examinando a importância relativa de diferentes características morfológicas para a variância fenotípica.

\section{MATERIAL E MÉTODOS}

Foram tomados dados do estipe, cacho, ráquilas, frutos e folhas de 129 plantas adultas de açaizeiro amostradas em ambiente antropizados de terra firme, como quintais caseiros e pequenas propriedades; e de várzeas, como margens de baías e rios. As matrizes foram coletadas em seis municípios e quatro microrregiões do nordeste paraense (Tabela 1), dando-se preferência às plantas em franca produção. O local de coleta foi determinado através do Sistema de Posicionamento Global-GPS.

Durante a coleta, tomaram-se dados de 22 caracteres morfoagronômicos quantitativos relativos à planta (5), aos frutos (6) e agronômicos (11). Os caracteres da planta foram: (1) Número de estipe por planta (NEP); (2) Altura do estipe mais velho da planta (AE, m); (3) Número de folhas existentes no estipe mais velho da planta (NF); (4) Circunferência do estipe mais velho da planta à altura do peito (CAP, $\mathrm{cm}$ ), e (5) Comprimento do entrenó (CEN, cm), referente a distância entre os nós (cicatrizes foliares) do estipe mais velho da planta, tendo como ponto de partida a altura do peito para baixo.

Os caracteres dos frutos foram avaliados em apenas um cacho da planta, sendo retirados, aleatoriamente, dez frutos. Os caracteres foram: (6) Diâmetro longitudinal do fruto (DLF, mm), medido em paquímetro digital com precisão de $0,02 \mathrm{~mm}$; (7) Diâmetro transversal do fruto (DTF, mm), medido em paquímetro digital, com precisão de $0,02 \mathrm{~mm}$; (8) Peso do fruto (PF, g), efetuado em balança de precisão; (9) Peso da semente (PS, g), obtido após a retirada da parte comestível (epicarpo e mesocarpo); (10) Peso da polpa (PP, g), calculado pela diferença entre os caracteres PF e PS; (11) Rendimento de polpa por fruto (RPF, \%), calculado pela razão entre os caracteres PP e PF, multiplicado por 100.

Os caracteres agronômicos foram: (12) Peso do cacho (PC, $\mathrm{kg}$ ), obtido pela média da pesagem de todos os cachos colhidos; (13) Peso de frutos por cacho (PFC, kg), obtido pela média da pesagem de 
todos os frutos produzidos por cacho; (14) Rendimento de frutos por cacho (RFC, \%), calculado pela razão entre os caracteres PFC e PC e multiplicado por 100; (15) Número de ráquilas por cacho (NRC), obtido pela média do número de ráquilas por cacho; (16) Número de frutos por ráquila (NFR), calculado pela média do número de frutos por ráquila; (17) Número de frutos por cacho (NFC), calculado pela média do número de frutos por cacho; (18) Número de cachos por planta (NCP), obtido pela contagem de todos os cachos da planta; (19) Peso de cem frutos (PCF, g), obtido pela média do peso de 100 frutos de todos os cachos por planta; (20) Circunferência do cacho (CIC, $\mathrm{cm}$ ), obtida pela média da circunferência de todos os cachos; (21) Comprimento total do cacho $(\mathrm{CC}, \mathrm{cm})$, obtido pela média do comprimento de todos os cachos, e (22) Produção total de frutos (PTF, kg), obtida pela pesagem de todos os frutos por planta.

Para a análise da variabilidade morfoagronômica, os dados das matrizes foram analisados por componentes principais. Para eliminar o efeito de diferentes escalas métricas no cálculo dos componentes principais, os valores foram padronizados pela transformação normal reduzida (Z). A identificação de caracteres redundantes foi feita, inicialmente, pela utilização do critério de descarte direto proposto por Jolliffe (1972, 1973) e Mardia et al. (1979), o qual preconiza iniciar pelos componentes que apresentam os menores autovalores até o limite de 0,70 associados aos maiores autovetores, nos respectivos componentes, e pelo método gradual de descarte proposto por Cury (1993), o qual recomenda que, a cada descarte, seja realizada uma nova análise com os caracteres remanescentes, observando a correlação do caráter redundante com os demais; o procedimento foi interrompido quando o caráter a ser descartado apresentou alta correlação com, no mínimo, um dos já descartados anteriormente. Porém, o descarte de cada caráter só ocorreu quando ele foi eliminado simultaneamente nos dois procedimentos.

A avaliação da eficiência do descarte realizou-se pela comparação de grupos de dissimilaridade das dez procedências de açaizeiro, com base nos 22 caracteres originais e nos 14 selecionados. Para a formação dos grupos, utilizou-se a distância euclidiana média e o método de otimização de Tocher modificado (VASCONCELOS et al., 2007). Utilizou-se, também, da análise da dispersão gráfica tridimensional, recomendada para complementar estudo baseado em componentes principais, em que os dois primeiros componentes não concentram, pelo menos, $80 \%$ da variabilidade (CRUZ; REGAZZI, 2008). As estimativas dos autovalores e autovetores, os coeficientes de correlação, a análise gráfica e a avaliação da eficiência do descarte foram obtidos no programa GENES (CRUZ, 2006).

\section{RESULTADOS E DISCUSSÃO}

$\mathrm{Na}$ Tabela 2, consta o resultado da técnica de análise de componentes principais, apresentando as estimativas dos autovalores associados aos 22 caracteres e suas respectivas variâncias absolutas, percentuais e acumuladas. Cada autovalor é uma fração da variância fenotípica total representada pelo respectivo caractere ordenado de maneira decrescente. A variância total foi de $22(100 \%)$, sendo que o primeiro componente respondeu por apenas $25,98 \%$ deste valor. Os dois, três, quatro, cinco e seis primeiros componentes acumularam, respectivamente, $42,09 \%, 52,78 \%, 61,29 \%, 68,01 \%$ e $73,59 \%$ da variância total.

Este resultado, apesar de próximo, é diferente do que foi encontrado por Oliveira et al. (2006) no estudo do germoplasma de açaizeiro para seleção de descritores que, ao analisarem 87 acessos, nos quais foram avaliados 28 descritores morfoagronômicos, obtiveram $35,8 \%$ da variação total nos dois primeiros componentes e $68,85 \%$ ficaram distribuídas até o sexto componente.

Os fatores que, provavelmente, mais contribuíram para as diferenças foi o fato de que dois caracteres aqui considerados não foram abordados por Oliveira et al. (2006) e oito, utilizados por estes autores, não foram incluídos neste estudo, devido à ausência de oportunidade para obtê-los, por se tratarem de dados de coleta; além disso, tais autores incluíram em seus estudos, acessos procedentes de outros estados da Federação, como Amapá e Maranhão, o que, em tese, deveria colaborar para maior variância fenotípica, porém não foi o que se observou, pois as matrizes procedentes do nordeste paraense apresentaram variação de 42,09 nos dois primeiros componentes, ao passo que acessos procedentes dos Estados do Pará, Amapá e Maranhão apresentaram $35,8 \%$ nos mesmos componentes.

A influência da distância geográfica sobre a variabilidade fenotípica pode ser corroborada em Manfio (2010), que, ao estudar agrupamentos de matrizes de palmeira macaúba com caracteres fenotípicos não selecionados por componentes principais, de diferentes Estados (MG, MS, SP, PE, MA e PA), detectou nove grupos divergentes. Se, neste estudo, fossem utilizados caracteres selecionados por componentes principais, então, muito provavelmente, seria obtido um número ainda maior de grupos.

Em outras palmeiras, foram encontrados percentuais na faixa de $33,86 \%$ a $41,63 \%$ nos dois 
primeiros componentes principais. Gomes (2007), com $41,63 \%$, em estudo sobre variabilidade fenotípica de caracteres vegetativos e reprodutivos em população de pupunheira (Bactris gasipaes Kunth), avaliou dez descritores em 1.978 plantas adultas do BAG de pupunheira do Instituto do Agronômico de Campinas (IAC), e Pinto et al. (2010), 33,86\%, em pesquisa sobre caracterização de 36 acessos de guariroba (Syagrus oleracea (Mart.) Becc.) para seleção de 18 descritores morfométricos.

$\mathrm{Na}$ análise de componentes principais, o ideal é que os dois primeiros componentes concentrem a maior quantidade de variância dos dados para que haja divergência entre grupos de genótipos (CRUZ; REGAZZI, 2008). Porém, a distribuição da variância está associada ao número e à natureza dos caracteres usados na análise, estando concentrada nos primeiros componentes, apenas quando se utiliza um número reduzido de descritores de interesse agronômico ou que estejam em um mesmo grupo, como folha, floração, frutos ou sementes, entre outros (PEREIRA et al., 1992). Ressalta-se, ainda, que, quando vários caracteres são altamente correlacionados, os primeiros componentes, tendem a explicar quase que a totalidade da variância total da nuvem de dados (FÁVERO et al., 2009). Gomes (2007), ao estudar nove descritores morfoagronômicos avaliados em frutos de 377 acessos do BAG - pupunheira - IAC, obteve $60,42 \%$.

$\mathrm{Na}$ Tabela 4, constam os caracteres préselecionados na seleção direta, seleção com reanálise e os selecionados para a caracterização de açaizeiro e, na Tabela 5, as estimativas dos coeficientes de correlação de Pearson entre os caracteres selecionados e os descartados.

O número de folhas, apesar de ter sido selecionado para descarte pelo critério de Cury, não foi excluído pelo critério de Jolliffe e também apresentou alta correlação com, no mínimo, um caráter já descartado anteriormente, além do que é o único caráter foliar adotado neste estudo. Diante disso, decidiu-se mantê-lo e encerrar o descarte na oitava reanálise.

Desta forma, foram descartados oito caracteres (PF, PFC, NFC, RPF, PCF, PTF, DTF e NFR). Observando os dois procedimentos, verifica-se que a seleção direta (método de Jolliffe) eliminou três dos cinco caracteres da planta e dez caracteres considerados importantes na avaliação da produção de frutos. A seleção com reanálise (método de Cury) mostrou-se menos rigorosa, excluindo-se somente os oito caracteres, que foram coincidentes com os já descartados na seleção direta.

Essas exclusões indicam que nenhum desses caracteres contribui substancialmente para a discriminação das matrizes, de modo que não necessitam ser coletados, proporcionando redução de trabalho, de tempo, de mão de obra e economia de recursos, os quais são elevados em espécies perenes, com perda insignificante para a caracterização (CRUZ et al., 2004).

Deste modo, a maior parte da variância fenotípica ficou contida nos 14 componentes ou caracteres, que, juntos, explicaram $97,61 \%$ da variação total, fazendo com que os componentes eliminados não afetassem significativamente a discriminação das matrizes. Dessa forma, sugere-se o descarte de 8 caracteres $(36,36 \%)$ para facilitar a caracterização e avaliação das matrizes de açaizeiro. Oliveira et al. (2006), analisando acessos de açaizeiro provenientes dos Estados do Pará, Amapá e Maranhão, excluíram $21,43 \%$ dos caracteres avaliados, diferença de resultados justificável, devido à utilização de materiais de diferentes municípios do Pará e de estados do Brasil, assim como de diferente natureza genética.

O descarte efetuado, portanto, não ocasionou perda significativa de informações, pois todos os caracteres redundantes apresentaram-se associados a, pelo menos, dois dos selecionados. Os três caracteres de frutos descartados, PF, RPF e DTF, são considerados importantes na avaliação e seleção de açaizeiros, porém o PF e o DTF estão altamente correlacionados aos caracteres selecionados DLF, PS, PP e PC, e o RPF está associado ao PS e ao PP. Oliveira et al. (2006) obtiveram também correlação entre os caracteres descartados PF e DTF aos caracteres selecionados DLF, PS e PC, mas não descartaram o RPF. Carvalho e Muller (2005), em estudo de caracterização biométrica e rendimento percentual de polpa de frutas nativas da Amazônia, verificaram que a variabilidade desse caráter em açaizeiro é baixa e bastante influenciada pelo ambiente.

Em relação aos caracteres agronômicos rejeitados, PFC, PTF, PCF também são citados como relevantes na seleção de frutos dessa espécie. Entretanto, alta correlação foi verificada entre PFC e PC e entre PTF e NCP. Oliveira et al. (2006) ratificam as relações aqui obtidas, inclusive ressaltando que o caráter PTF pode ser explicado totalmente pelo NCP.

Quanto ao PF, tal como o PCF, foi correlacionado aos caracteres DLF, PC, PS e PP. No entanto, Rogez (2000) relata, em revisão sobre o açaí, que o caráter PF não possui nenhuma relação com teor de matéria seca (PP), responsável pelo aumento do rendimento de açaí.

Sobre os outros caracteres agronômicos descartados, NFC e NFR estão altamente correlacionados ao caráter selecionado PC. Oliveira e Fernandes 
(2001) realizaram estudo para obtenção de estimativas do coeficiente de repetibilidade em caracteres do cacho de açaizeiro, como o NFC, para determinar a variabilidade genética em 30 genótipos, a análise da variância apresentou diferença significativa entre os genótipos $(\mathrm{P}<0,001)$, porém o coeficiente de repetibilidade foi inexpressivo, indicando que esse caráter é altamente influenciado pelo ambiente.

Em referência aos caracteres selecionados neste trabalho, Oliveira et al. (2006) destacaram a importância de 12 deles (NEP, AE, NF, CAP, CEN, DLF, PS, NCP, PC, RFC, NRC e CC) para discriminação de acessos de açaizeiro, com exceção apenas do rendimento de polpa por fruto. Considerando-se os seis primeiros componentes principais, os caracteres selecionados que mais contribuíram para a representação das matrizes foi, em ordem decrescente: PC, RFC, NRC, DLF, NF, NCP, AE, CAP, CIC, CC, CEN, NEP, PS e PP. Os caracteres número de estipes em frutificação por planta, comprimento da bainha foliar, dias de floração masculina, intervalo entre fases de floração, dias de floração feminina, dias após a polinização, número de anos em produção e número de meses em produção mencionados no trabalho desses autores não foram considerados neste estudo por se tratar de dados de coleta. Entretanto, foram estudados os caracteres como número de frutos por ráquila, número de frutos por cacho, circunferência do cacho e comprimento do cacho.

A dispersão gráfica dos três primeiros componentes principais se encontra na Figura 1. De modo geral, não se observou tendência de formação de agrupamentos na dispersão gráfica com base nos três primeiros componentes principais. No entanto, algumas matrizes destacaram-se por estarem relativamente separadas do conjunto, como é o caso dos pontos dez (EO-010 - Belém - BelémPA), e 13 (EO-013 - São João de Pirabas - Região do Salgado), 51 (EO-051 - Capitão Poço - Região do Guamá) e 18 (EO-018 - Salinópolis - Região do Salgado), esta se destacou pelo menor nível de similaridade. As matrizes EO-018 e EO-035 foram as mais divergentes. As demais formaram um único agrupamento continuamente distribuído no espaço, com alta similaridade morfológica, porém apresentou, na parte central, maior compactação de matrizes, podendo ser considerado como um subgrupo, sendo que este grupo vai descompactando-se tanto para o lado direito quanto para o esquerdo, culminando nas matrizes separadas do conjunto.

Percebeu-se, também, que, em relação ao plano lateral e ao plano de fundo, as matrizes EO124 e EO-111 (Ilha do Combu), EO-034 e EO-035 (Capitão Poço) e EO-022 (Santarém Novo) ficaram acima da média do grupo central. No extremo oposto, surgiram as matrizes EO-020 (Salinópolis), EO-054 (Ilha do Combu) e outras não claramente perceptíveis, presentes no subgrupo de compactação, sobre o plano basal. Portanto, em relação à média, apresentaram parcial divergência, as procedências da Ilha do Combu, Capitão Poço e Santarém Novo (matrizes mais acima da média) com a procedência de Salinópolis e a procedência da Ilha do Combu.

Em estudo sobre caracterização morfológica de 40 matrizes de pimenta-de-macaco (Piper aduncum L. - autógama), a variação distribuiu-se nos sete primeiros componentes principais, apresentando complementação com a dispersão gráfica 3D semelhante às 129 matrizes de açaizeiro (alógama), embora com menor compactação de matrizes em relação ao plano basal, formou um grupo relativamente homogêneo e contínuo, porém, como nestas matrizes de açaizeiro, permitiu detectar divergência genética (GAIA et al., 2011).

Em açaizeiro do Banco de Germoplasma da EMBRAPA - Amazônia Oriental, o estudo de acessos mostrou que a variância se acumulou nos nove primeiros componentes principais, não havendo complementação gráfica 3D. Em espécies frutíferas, como o pessegueiro (Prunus persica), o estudo sobre distância genética, em populações de cruzamento dirigido, verificou que os três primeiros componentes foram suficientes para explicar mais de $80 \%$ da variação total (WAGNER JÚNIOR et al., 2011), nível de variação suficiente para identificar grupos divergentes, fundamentais para o melhoramento genético das espécies.

Foram comparados os grupos de dissimilaridade das 10 procedências de açaizeiro, com base nos 22 caracteres originais e nos 14 selecionados das 129 matrizes; para a formação dos grupos, utilizaram-se a distância euclidiana média e o método de otimização de Tocher modificado para avaliar a eficiência de descarte (Tabela 6). Verificou-se que, no agrupamento das procedências pelos caracteres originais, foram obtidos três grupos distintos: o primeiro, constituído de oito procedências, o segundo e o terceiro apenas de uma procedência. Utilizando os 14 caracteres selecionados, foram obtidos quatro grupos, com diferenças em relação à distribuição e ao número de genótipos nos grupos (Tabela 6). A procedência de Belém ocupou um grupo separado, tanto utilizando 22 como 14 caracteres.

Os resultados mostraram $60 \%$ de coincidência das procedências no maior grupo de similaridade. Os grupos formados pelos 14 caracteres selecionados tiveram melhor discriminação, considerando a separação das procedências de Ilha de Mosqueiro, 
Salinas e Ourém.

Oliveira et al. (2006), comparando grupos de dissimilaridade pelo método de Tocher nos descritores originais, observaram a formação de quatro grupos, e nos 22 selecionados foram discriminados sete grupos, havendo modificações em relação ao número e à distribuição, com coincidência total no último grupo e parcial no penúltimo e último grupos; e concluíram que a maior eficiência ocorreu com os caracteres selecionados devido à formação de mais grupos, com acréscimo de $75 \%$. Isto se deve, provavelmente, ao fato de que os acessos analisados por esses autores não foram somente procedentes do Estado do Pará, ao passo que as matrizes aqui estudadas se restringem a municípios do nordeste paraense, próximos a Belém, o que, provavelmente, contribuiu para um acréscimo de somente $33,33 \%$ no agrupamento formado pelos caracteres selecionados.

TABELA 1 - Caracterização de 129 matrizes de açaizeiro quanto à microrregião, local, ponto de coleta, posicionamento global e ao clima, amostradas no nordeste paraense. Belém-PA, UFRA, 2011.

\begin{tabular}{|c|c|c|c|c|c|}
\hline Matrizes & NM & Local & Ponto de coleta & Fuso/P & Clima \\
\hline EO-001 & 1 & BEL Belém & Tv. Lomas & $22 / \mathrm{E}=785321,519 / \mathrm{N}=9839863,229$ & Afi \\
\hline EO-002 a EO-007 & 6 & BEL Ilha de Mosqueiro & Baía do Sol & $22 / E=786327,535 / N=4941319,702$ & Afi \\
\hline EO-008 a EO-010 & 3 & BEL Belém & UFRA & $22 / \mathrm{E}=785178,499 / \mathrm{N}=9839169,524$ & Afi \\
\hline EO-011 a EO-014 & 4 & RSA São João de Pirabas & sBoa esperança & $23 / \mathrm{E}=253649,066 / \mathrm{N}=9991304,549$ & Awi \\
\hline EO-015 a EO-021 & 7 & RSA Salinópolis & Vila de São Bento & $23 / \mathrm{E}=240441,851 / \mathrm{N}=9923125,540$ & Awi \\
\hline EO-022 a EO-028 & 7 & ZBR Santarém Novo & $\begin{array}{l}\text { Vila de São João } \\
\text { de Peri Meri: }\end{array}$ & $23 / \mathrm{E}=240934,783 / \mathrm{N}=9901884,077$ & Ami \\
\hline EO-029 a EO-032 & 4 & RSA São João de Pirabas & sRua Paricá & $23 / E=255961,817 / N=9910957,315$ & Awi \\
\hline EO-033 a EO-040 & 8 & RGU Capitão Poço & Bairro Timbó & $23 / E=270732,801 / N=9809916,910$ & Ami \\
\hline EO-041 a EO-043 & 3 & RGU Ourém & Tv. Cipriano Santos & $23 / \mathrm{E}=264406,104 / \mathrm{N}=9828590,344$ & Ami \\
\hline EO-044 a EO-053 & 10 & RGU Capitão Poço & Bairro Cotilandia & $23 / E=269593,245 / N=9805952,361$ & Ami \\
\hline EO-054 a EO-094 & 41 & BEL Ilha do Combu & Beira rio & $22 / \mathrm{E}=781177,000 / \mathrm{N}=9834854,000$ & Afi \\
\hline EO-095 a EO-107 & 13 & BEL Ilha do Combu & Furo São Sebastião & $22 / \mathrm{E}=779499,000 / \mathrm{N}=9833506,000$ & Afi \\
\hline EO-108 a EO-129 & 22 & BEL Ilha do Combu & Rio Jordão & $22 / \mathrm{E}=784013,000 / \mathrm{N}=9832788,000$ & Afi \\
\hline
\end{tabular}

NM: número de matrizes; MR: Microrregião; FUSO/P: Fuso/Posição no GPS; BEL: Belém; RSA: Região do Salgado; ZBR: Zona Bragantina; RGU: Região do Guamá.

TABELA 2 - Estimativa dos autovalores associados aos componentes principais e suas variâncias percentual e acumulada dos 22 caracteres morfoagronômicos, em 129 matrizes de açaizeiro. Belém-PA, UFRA, 2011.

\begin{tabular}{cccc}
\hline Componente & Autovalor & Variância (\%) & Variância acumulada \\
\hline $\mathbf{1}$ & 5,7152 & 25,98 & 25,98 \\
$\mathbf{2}$ & 3,5431 & 16,11 & 42,08 \\
$\mathbf{3}$ & 2,3514 & 10,69 & 52,77 \\
$\mathbf{4}$ & 1,8731 & 8,51 & 61,29 \\
$\mathbf{5}$ & 1,4777 & 6,72 & 68,00 \\
$\mathbf{6}$ & 1,2277 & 5,58 & 73,58 \\
$\mathbf{7}$ & 1,0977 & 4,99 & 78,57 \\
$\mathbf{8}$ & 0,8559 & 3,89 & 82,46 \\
$\mathbf{9}$ & 0,7434 & 3,38 & 85,84 \\
$\mathbf{1 0}$ & 0,6256 & 2,84 & 88,69 \\
$\mathbf{1 1}$ & 0,5840 & 2,65 & 91,34 \\
$\mathbf{1 2}$ & 0,5222 & 2,37 & 93,71 \\
$\mathbf{1 3}$ & 0,4772 & 2,17 & 95,88 \\
$\mathbf{1 4}$ & 0,3791 & 1,72 & 97,61 \\
$\mathbf{1 5}$ & 0,2334 & 1,06 & 98,67 \\
$\mathbf{1 6}$ & 0,1734 & 0,79 & 99,46 \\
$\mathbf{1 7}$ & 0,0534 & 0,24 & 99,70 \\
$\mathbf{1 8}$ & 0,0485 & 0,22 & 99,92 \\
$\mathbf{1 9}$ & 0,0093 & 0,04 & 99,96 \\
$\mathbf{2 0}$ & 0,0053 & 0,02 & 99,98 \\
$\mathbf{2 1}$ & 0,0033 & 0,02 & 100,00 \\
$\mathbf{2 2}$ & 0,0001 & 0,00 & 100,00 \\
\hline
\end{tabular}




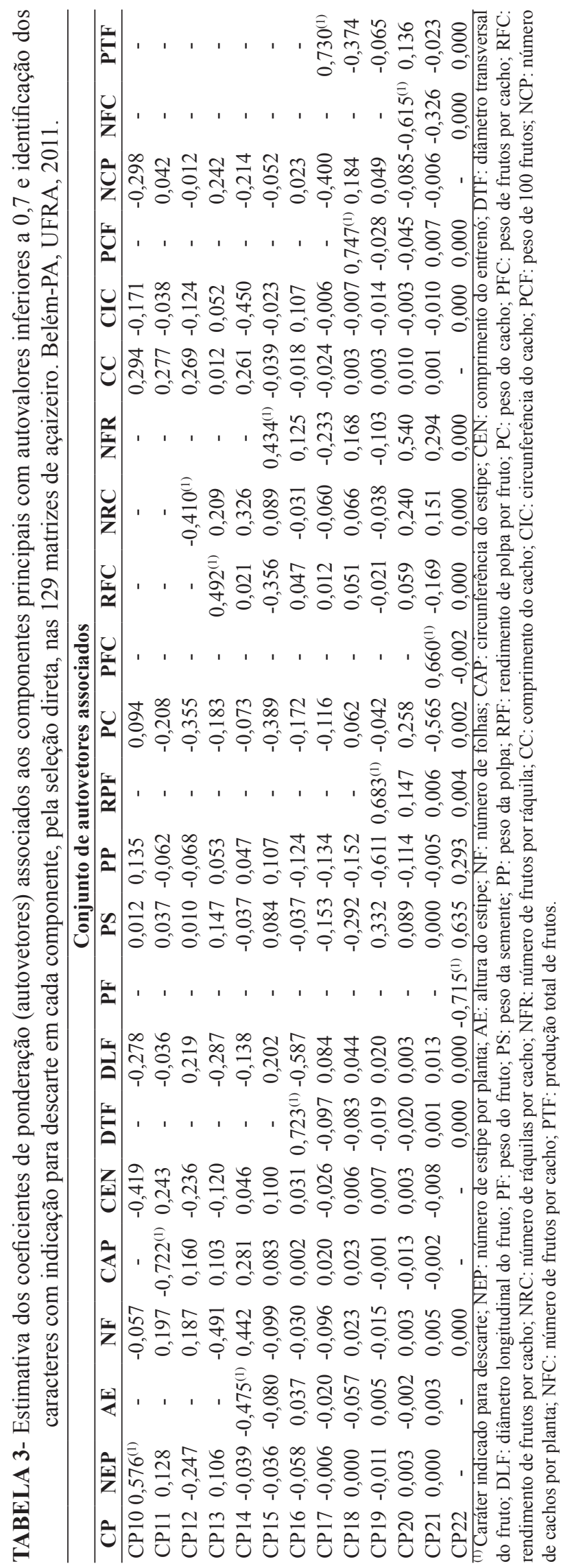


TABELA 4- Caracteres pré-selecionados nos procedimentos de seleção direta e seleção com reanálise e os selecionados para a caracterização de açaizeiro. Belém-PA, UFRA, 2011.

\begin{tabular}{cccc}
\hline & \multicolumn{2}{c}{ Pré-selecionados } \\
\cline { 2 - 3 } Caracteres & \multicolumn{2}{c}{ Selecionados } \\
\cline { 2 - 3 } NEP & Método de Jolliffe e Mardia & Método de Cury \\
AE & NPSEL & PSEL & SEL \\
NF & NPSEL & PSEL & SEL \\
CAP & PSEL & NPSEL $\left(9^{\circ}\right)$ & SEL \\
CEN & NPSEL & PSEL & SEL \\
DTF & PSEL & PSEL & SEL \\
DLF & NPSEL $\left(8^{\circ}\right)$ & NPSEL $\left(8^{\circ}\right)$ & NSEL \\
PF & PSEL & PSEL & SEL \\
PS & NPSEL $\left(1^{\circ}\right)$ & NPSEL $\left(1^{\circ}\right)$ & NSEL \\
PP & PSEL & PSEL & SEL \\
RPF & PSEL & PSEL & SEL \\
PC & NPSEL $\left(4^{\circ}\right)$ & NPSEL $\left(4^{\circ}\right)$ & NSEL \\
PFC & PSEL & PSEL & SEL \\
RFC & NPSEL $\left(2^{\circ}\right)$ & NPSEL $\left(2^{\circ}\right)$ & NSEL \\
NRC & NPSEL & PSEL & SEL \\
NFR & NPSEL & PSEL & SEL \\
CC & NPSEL $\left(7^{\circ}\right)$ & NPSEL $\left(7^{\circ}\right)$ & NSEL \\
CIC & PSEL & PSEL & SEL \\
PCF & PSEL & PSEL & SEL \\
NCP & NPSEL $\left(5^{\circ}\right)$ & NPSEL $\left(5^{\circ}\right)$ & NSEL \\
NFC & PSEL & PSEL & SEL \\
PTF & NPSEL $\left(3^{\circ}\right)$ & NPSEL $\left(3^{\circ}\right)$ & NSEL \\
\hline
\end{tabular}

NEP: número de estipe por planta; AE: altura do estipe; NF: número de folhas; CAP: circunferência do estipe; CEN: comprimento do entrenó; DTF: diâmetro transversal do fruto; DLF: diâmetro longitudinal do fruto; PF: peso do fruto; PS: peso da semente; PP: peso da polpa; RPF: rendimento de polpa por fruto; PC: peso do cacho; PFC: peso de frutos por cacho; RFC: rendimento de frutos por cacho; NRC: número de ráquilas por cacho; NFR: número de frutos por ráquila; $\mathrm{CC}$ : comprimento do cacho; CIC: circunferência do cacho; PCF: peso de 100 frutos; NCP: número de cachos por planta; NFC: número de frutos por cacho; PTF: produção total de frutos.

TABELA 5 - Estimativas dos coeficientes de correlação de Pearson entre os caracteres morfoagronômicos selecionados e os descartados avaliados, em 129 matrizes de açaizeiro. Belém, UFRA, 2011.

\begin{tabular}{clllllllll}
\hline \multirow{2}{*}{ Selecionados (1) } & \multicolumn{10}{c}{ PF } & \multicolumn{1}{c}{ PFC } & \multicolumn{1}{c}{ NFC } & \multicolumn{1}{c}{ RPF } & \multicolumn{1}{c}{ PCF } & \multicolumn{1}{c}{ PTF } & \multicolumn{1}{c}{ DTF } & NFR \\
\hline NEP & 0,020 & $-0,025$ & $-0,044$ & 0,004 & 0,027 & 0,077 & $0,255^{* *}$ & 0,004 \\
AE & 0,073 & 0,037 & 0,118 & $-0,084$ & 0,100 & $0,213^{*}$ & $-0,145$ & $-0,061$ \\
NF & $0,197^{*}$ & $0,414^{* *}$ & $0,434^{* *}$ & 0,031 & $0,187^{*}$ & $0,879^{* *}$ & 0,080 & $0,378^{* *}$ \\
CAP & $-0,094$ & 0,153 & 0,141 & $-0,152$ & 0,073 & 0,077 & $-0,009$ & 0,016 \\
CEN & 0,024 & 0,117 & 0,041 & 0,061 & $-0,029$ & $0,268^{* *}$ & 0,033 & 0,060 \\
DLF & $0,813^{* *}$ & $0,322^{* *}$ & 0,166 & 0,041 & $0,384^{* *}$ & $0,301 * *$ & $0,881 * *$ & 0,136 \\
PS & $0,911^{* *}$ & $0,185^{*}$ & $-0,032$ & $-0,449^{* *}$ & $0,873^{* *}$ & $0,193^{*}$ & $0,432^{* *}$ & $-0,116$ \\
PP & $0,465^{* *}$ & 0,114 & $-0,042$ & $0,855^{* *}$ & $0,440^{* *}$ & 0,155 & $0,392^{* *}$ & $-0,048$ \\
PC & $0,202^{*}$ & $0,969^{* *}$ & $0,769^{* *}$ & $-0,062$ & 0,171 & $0,727^{* *}$ & $0,278^{* *}$ & $0,730^{* *}$ \\
RFC & 0,160 & $0,625^{* *}$ & $0,595^{* *}$ & 0,166 & 0,090 & $0,527^{* *}$ & $0,264^{* *}$ & $0,561 * *$ \\
NRC & 0,140 & $0,308^{* *}$ & $0,432^{* *}$ & $-0,107$ & 0,118 & $0,245^{* *}$ & 0,103 & 0,035 \\
CC & 0,095 & $0,221^{*}$ & 0,169 & $-0,002$ & 0,093 & 0,134 & 0,079 & 0,129 \\
CIC & 0,056 & $-0,042$ & $-0,026$ & 0,117 & 0,049 & $-0,103$ & $-0,031$ & $-0,150$ \\
NCP & 0,046 & $-0,086$ & $0,257^{* *}$ & 0,130 & 0,043 & $0,868^{* *}$ & 0,168 & $0,301^{* *}$ \\
\hline
\end{tabular}

(1):NEP: número de estipe por planta; AE: altura do estipe; NF: número de folhas; CAP: circunferência do estipe; CEN: comprimento do entrenó; DLF: diâmetro longitudinal do fruto; PS: peso da semente; PP: peso da polpa; PC: peso do cacho; RFC: rendimento de frutos por cacho; NRC: número de ráquilas por cacho; CC: comprimento do cacho; CIC: circunferência do cacho; NCP: número de cachos por planta; (2):PF: peso do fruto; PFC: peso de frutos por cacho; NFC: número de frutos por cacho; RPF: rendimento de frutos por cacho; PCF: peso de 100 frutos; PTF: produção total de frutos; DTF: diâmetro transversal do fruto; NFR: número de frutos por ráquila. 
TABELA 6 - Grupos de dissimilaridade constituídos de 10 procedências de açaizeiro formados pelo método de Tocher, das 129 matrizes, com base nos 22 caracteres originais e nos 14 selecionados. Belém-PA, UFRA, 2011.

\begin{tabular}{ccc}
\hline \multirow{2}{*}{ Grupo } & \multicolumn{2}{c}{ Procedência } \\
\cline { 2 - 3 } 22 caracteres originais & $\mathbf{1 4}$ caracteres selecionados \\
\hline & Ilha de Mosqueiro, São João de Pirabas, & Santarém Novo, Ourém, \\
1 & Ilha do Combu (Rio Jordão), Salinas, & Sa de Pirabas, Santarém Novo, \\
Ilha do Combu (Furo São Sebastião), & Ilha do Combu (Furo São Sebastião), \\
& Ilha do Combu (Beira-rio) & Ilha do Combu (Rio Jordão) \\
3 & Capitão Poço & Ilha de Mosqueiro e Salinas \\
4 & Belém & Belém \\
& & Ourém \\
\hline
\end{tabular}

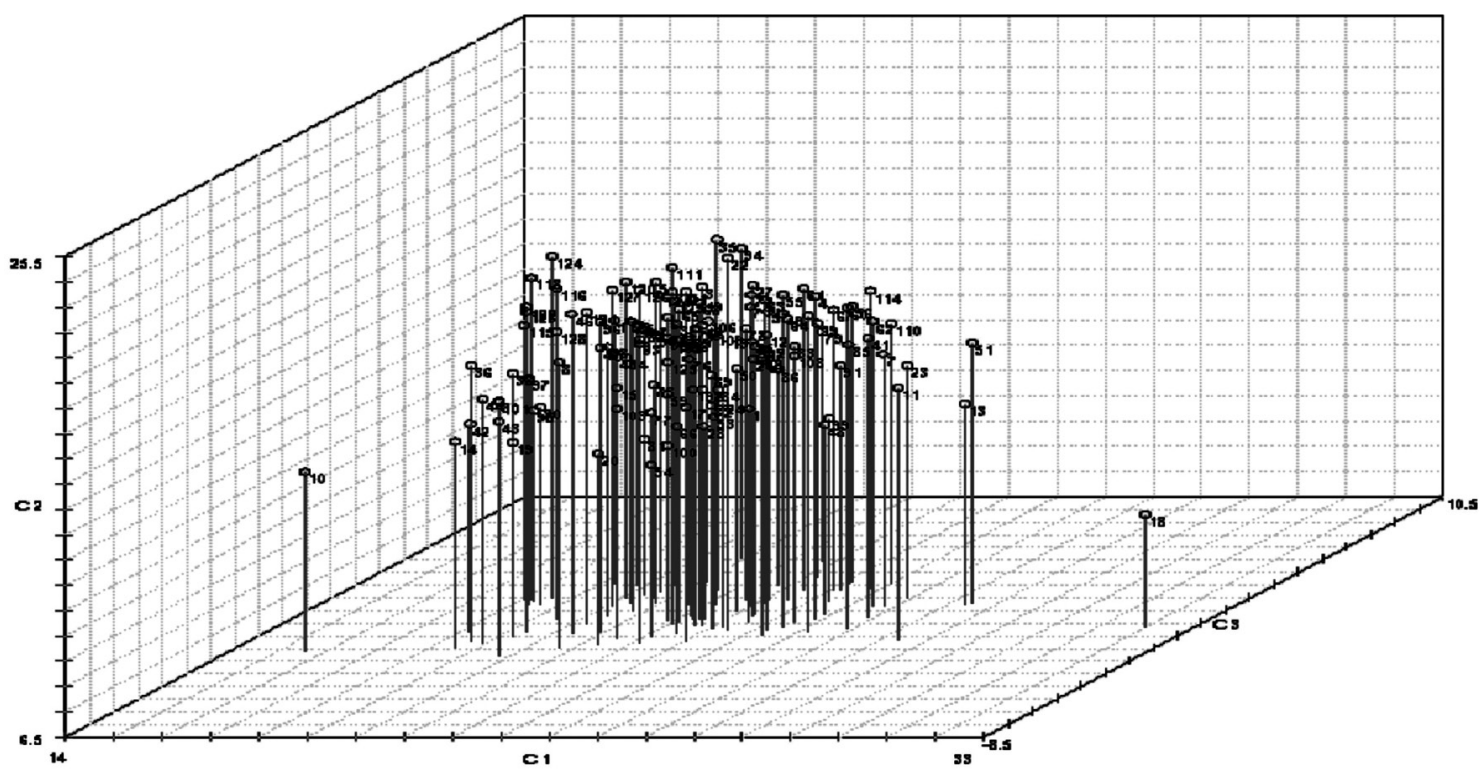

FIGURA 1 - Dispersão gráfica 3D dos primeiros componentes principais com base em 22 caracteres morfoagronômicos de açaizeiro. Belém, UFRA, 2011.

\section{CONCLUSÃO}

Conclui-se que os genótipos de açaizeiro aqui estudados podem ser discriminados, com perda mínima de variância, pelos caracteres NEP, AE, CAP, CEN, NF (caule e folha), DLF, PS, PP, PC, RFC, NRC, CC, CIC e NCP (semente, fruto e cacho), havendo uma redução de $36,4 \%$ em relação ao número inicialmente proposto para caracterizar este germoplasma, apresentando matrizes divergentes, importantes para aumentar a variabilidade genética da coleção e o desenvolvimento de genótipos superiores.

\section{REFERÊNCIAS}

ALVES, R.M.; GARCIA A.A.F; CRUZ, E.D.; FIGUEIRA, A. Seleção de descritores botânicoagronômicos para caracterização de germoplasma de cupuaçuzeiro. Pesquisa Agropecuária Brasileira, Brasília, v.38, n.7, p.807-818, 2003.

CARVALHO, J.E.U.; MÜLLER, C.H. Biometria e rendimento percentual de polpa de frutas nativas da Amazônia. Belém: Embrapa Amazônia Oriental, 2005. 3p. (Comunicado Técnico, 139). 
CARVALHO, J.E.U.; NASCIMENTO, W.M.O.; MÜLLER, C.H. Características físicas e germinativas de sementes de espécies frutíferas nativas da Amazônia. Belém: EMBRAPA-CPATU, 1998. p.1-18. (Boletim de Pesquisa, 203).

CRUZ, C.D. Programa Genes: análise multivariada e simulação. Viçosa: Editora UFV, 2006. 175p.

CRUZ, C.D.; REGAZZI, A.J. Modelos biométricos aplicados ao melhoramento genético. 2.ed. Viçosa: UFV, 2008. 390p.

CRUZ, C.D.; REGAZZI, J.A.; CARNEIRO, P.C.S. Modelos biométricos aplicados ao melhoramento genético. Viçosa: UFV, 2004. v.1, p.377-413.

CURY, R. Dinâmica evolutiva e caracterização de germoplasma de mandioca (Manihot esculenta, Crantz) na agricultura autóctone do sul do Estado de São Paulo. 1993. 103 f. Dissertação (Mestrado) - Escola Superior de Agricultura Luiz de Queiroz, Universidade de São Paulo, Piracicaba, 1993.

DAHER, R.F.; MORAES, C.F.; CRUZ, C.D. Seleção de caracteres morfológicos em capim-elefante (Pennisetum purpureum Schum.). Revista Brasileira de Zootecnia, Viçosa, MG, v.26, n.2, p.247-259, 1997.

DIAS, L.A.S.; KAGEYAMA, P.Y.; CASTRO, G.C.T. Divergência genética multivariada na preservação de germoplasma de cacau (Theobroma cacao L.). Agrotrópica, Itabuna, v.9, n.1, p.29-40, 1997.

FÁVERO, L.P.; BELFIORE, P.; SILVA, F.L.; CHAN, B.L. Análise de dados: modelagem multivariada para tomada de decisões. Rio de Janeiro: Campus/ Elsevier, 2009. 646p.

GAIA, J.M.D.; MOTA, M.G.C.; CONCEIÇÃO, C.C.C.; MAIA, J.G.S. Morphologic characterization of spiked pepper's germplasm. Horticultura Brasileira, Brasília, v.29, n.2, 162-167, 2011.

GOMES, D.M. Variabilidade fenotípica de caracteres vegetativos e reprodutivos em população de pupunheira (Bactris gasipaes Kunth). 2007. 72f. Dissertação (Mestrado em Agricultura Tropical e Subtropical) - Instituto Agronômico de Campinas, Campinas, 2007.
GONÇALVES, A.A.S. Crescimento, composição mineral e sintomas visuais de deficiências de macronutrientes e boro em plantas de açaizeiro (Euterpe oleracea Mart.). Belém, 2004. 161f. Dissertação (Mestrado em Agronomia) - Universidade Federal Rural da Amazônia, Pará, 2004.

JOLLIFFE, I.T. Discarding variables in a principal component analysis; I. Artificial data. Applied Statistics, London, v.21, n.2, p.160-173, 1972.

JOLLIFFE, I.T. Discarding variables in a principal component analysis; II. Real data. Applied Statistics, London, v.22, n.1, p.21-31, 1973.

MANFIO, C.E. Análise genética no melhoramento da macaúba. 2010.52 f. Tese (Doutorado) - Universidade Federal de Viçosa, Viçosa, 2010..

MARDIA, K.L.; KENT, J.T.; BIBBY, J.M. Multivariate analysis. London: Academic Press, 1979. $521 \mathrm{p}$.

MENEZESNETO, M.A.;ALVES, J.D.; OLIVEIRA, L.E.M. Anaerobic metabolism of Euterpe oleracea. II. Plant tolerance to anoxia. Revista Brasileira de Fisiologia Vegetal, Londrina, v.7, n.1, p.47-51, 1995.

OLIVEIRA, M.S.P.; FERNANDES, G.L.C. Repetibilidade de caracteres do cacho de açaizeiro nas condições de Belém-PA. Revista Brasileira de Fruticultura, Jaboticabal, v.23, n.3, p.613-616, 2001.

OLIVEIRA, M.S.P.; FERREIRA, D.F.; SANTOS, J.B. Seleção de descritores para caracterização de germoplasma de açaizeiro para produção de frutos. Pesquisa Agropecuária Brasileira, Brasília, v.41, n.7, p.1133-1140, 2006.

PEREIRA, A.V.; VENCOVSKY, R.; CRUZ, C.D. Selection of botanical and agronomical descriptors for the characterization of cassava (Manihot esculenta Crantz.) germplasm. Revista Brasileira de Genética, Ribeirão Preto, v.15, n.1, p.115-124, 1992.

PINTO, J.F.N.; REIS, E.F.; FALEIRO, F.G.; BARBOSA, E.C.C.; NUNES, H.F.; PINTO, J.F.N. Seleção de descritores vegetativos para caracterização de acessos de guariroba (Syagrus oleracea (Mart.) Becc.). Revista Brasileira de Fruticultura, Jaboticabal, v.32, n.3, p.1-8, 2010. 
ROGEZ, H. Açaí: preparo, composição e melhoramento da conservação. Belém: EDUFPA, 2000. 313p.

SANTANA, A.C.; CARVALHO, D.F.; MENDES, F.A.T. Análise Sistêmica da fruticultura paraense: organização, mercado e competitividade empresarial. Belém: Banco da Amazônia, 2008. 255p.

STRAPASSON, E.; VENCOVSKY, R.; BATISTA, L.A.R. Seleção de descritores na caracterização de germoplasma de Paspalum sp por meio de componentes principais. Revista Brasileira de Zootecnia, Viçosa, MG, v.29, n.2, p.373-381, 2000
VASCONCELOS, E.S.; CRUZ, C.D.; BHERING, L.L.; RESENDE JÚNIOR, M.F.R. Método alternativo para análise de agrupamento. Pesquisa Agropecuária Brasileira, Brasília, v.42, n.10, p.1421-1428, out. 2007.

WAGNER JÚNIOR, A.; BRUCKNER, C.H.; CANTÍN, C.M.; SÁNCHEZ, M.A.M.; CRUZ, C.D. Divergência genética entre progênies de pessegueiro em Zaragoza, Espanha. Revista Brasileira de Fruticultura, Jaboticabal, v.33, n.1, p.303-310, 2011. 